\title{
Irradiance Variations of Stars
}

\author{
By G. WESLEY L O C K W O O D
}

Lowell Observatory, 1400 West Mars Hill Road, Flagstaff, AZ 86001, USA

Using a nine-year time series of photometric observations of 33 stars similar in temperature and mass to the Sun, but covering a wide range of age and mean chromospheric activity, we found that a majority varies from year to year, some by as much as several percent. We describe the methodology, circumstances of the observations, and photometric results. Stars most similar in mean chromospheric activity to the Sun varied by amounts several times greater than the Sun over a comparable time interval. Thus, the Sun's present low level of variability, as measured from 1980 to 1989 by the Solar Maximum Mission, appears unusual.

\section{Introduction}

Throughout the early decades of this century, a number of scientists were convinced that the solar constant varied by up to several percent and that these variations were associated with fluctuations in the Earth's climate (e.g., Clayton 1923; Huntington 1923; Abbott 1963). This enduring if elusive idea has been explored anew in recent conferences (e.g., McCormac 1983; Schatten \& Arking 1990).

Intrigued by measurements of the solar constant which seemed to leave open the question of solar variability, astronomers at the Lowell Observatory began monitoring the steadiness of the sunlight reflected from the planets Uranus and Neptune in 1949. The Air Force Cambridge Research Laboratory sponsored this study until 1966 with the goal of improving weather forecasts by uncovering the supposed connection to solar variability.

By comparing the brightness of the planets with stars nearby in the sky, making differential rather than absolute measurements, the Lowell observers avoided the worst complications of direct solar observation-the variability and spectral composition of the transmission of the Earth's atmosphere, and the need for an absolutely calibrated (and stable!) radiometer. They found no plausible evidence for intrinsic variations in planetary brightness attributable to solar changes. However, their related studies of solartype stars provided benchmark stellar data for the ongoing quest for solar variations. To standardize the planetary photometry, they carefully measured a small group of 16 solartype stars many nights each year from 1955 to 1966. In a report summarizing their results, Jerzykiewicz \& Serkowski (1966) concluded that “. . . this long series of photoelectric observations has taught us more about the variations of solar-type stars than about the Sun itself. The observations . . . indicate that for none of these stars does the standard deviation of the yearly mean magnitude exceed 0.008 , and for [three stars] this deviation is less than 0.004 mag."

Their observations thus capped the possible variability of sunlike stars at the $1 \%$ level but left open the question of smaller fluctuations. Our recent work reveals a rich domain of variability below $1 \%$ populated by stars whose photometric behavior mimics the solar paradigm in exaggerated form.

\section{Differential photometric observations of solar-type stars}

With vastly improved equipment, we undertook a photometric study of sunlike stars in 1981, using an 0.5-m reflecting telescope reserved for long-term photometric programs. At first, we concentrated on young, active stars in the Hyades open cluster. To our 


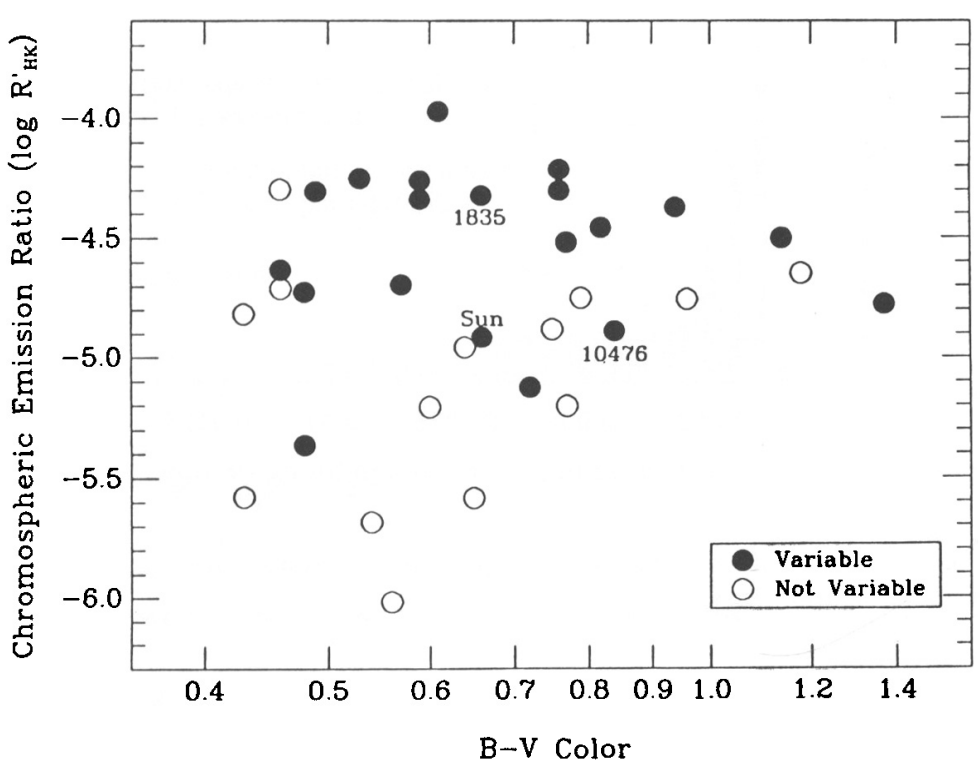

Figure 1. Properties of the program stars and the Sun. The symbols indicate whether we have found the stars to be variable or not. The ordinate, $\log \mathrm{R}_{\mathrm{HK}}^{\prime}$, the mean chromospheric emission ratio, is an estimate of the fraction of the stars' total output appearing as chromospheric emission (Noyes et al. 1984). The $B--V$ color, expressed as the magnitude difference between broadband filters at 0.44 and 0.55 microns, is a measure of photospheric temperature.

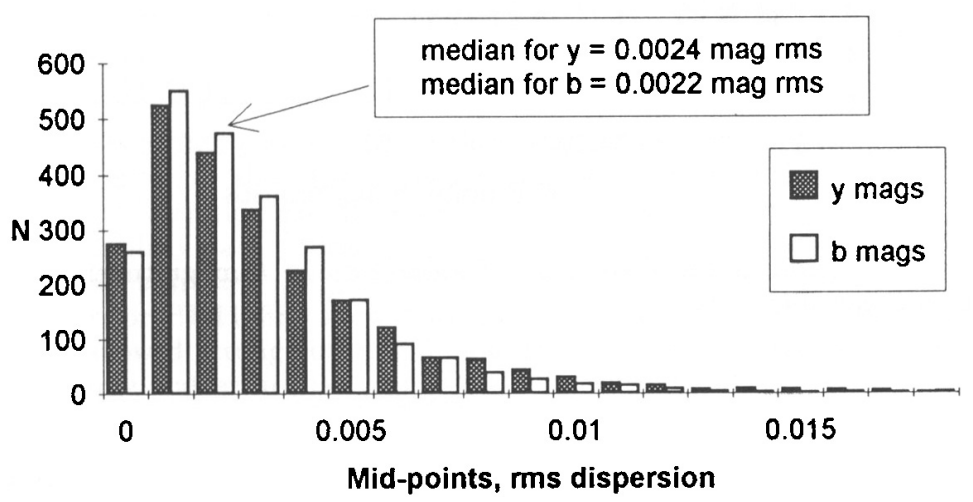

FIgURE 2. Distribution of the repeatability of differential $b$ and $y$ measurements made in a $y, b, b, y$ sequence.

surprise, we found that many were variable by up to several percent from night to night and from year to year (Radick et al. 1987). Further, brightness and Ca II emission are inversely correlated, strongly suggesting an association with active regions.

In 1984, we began measuring ordinary main sequence field stars, concentrating on 33 F, G, and $\mathrm{K}$ type dwarf and subgiant stars originally observed in $\mathrm{Ca}$ II $\mathrm{H}$ and $\mathrm{K}$ at Mount Wilson Observatory (Wilson 1978). These stars bracket the Sun in age, mass, temperature, and mean chromospheric activity (Figure 1). Some show cyclic activity mimicking the Sun (Baliunas \& Vaughan 1985); others appear quiescent, perhaps in Maunder minima (Baliunas \& Jastrow 1990). 


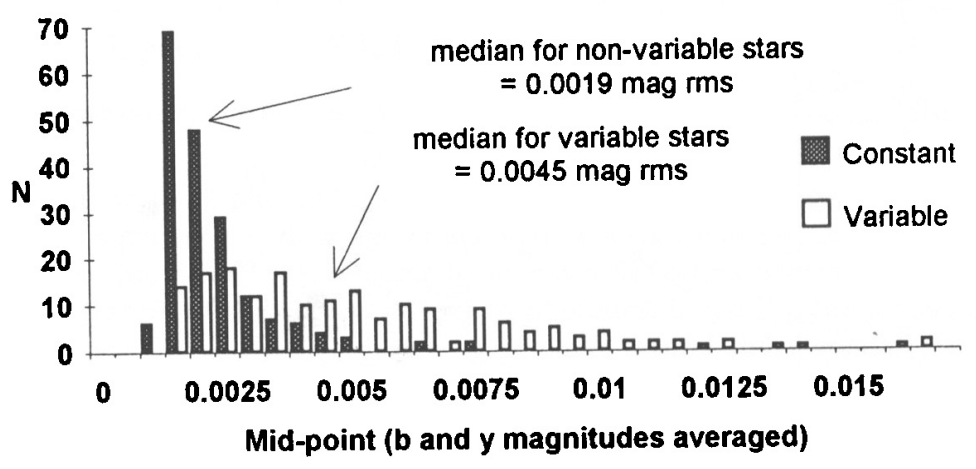

FIgURE 3. Distribution of the observed magnitude dispersion over an observing season for non-variable and variable stars.

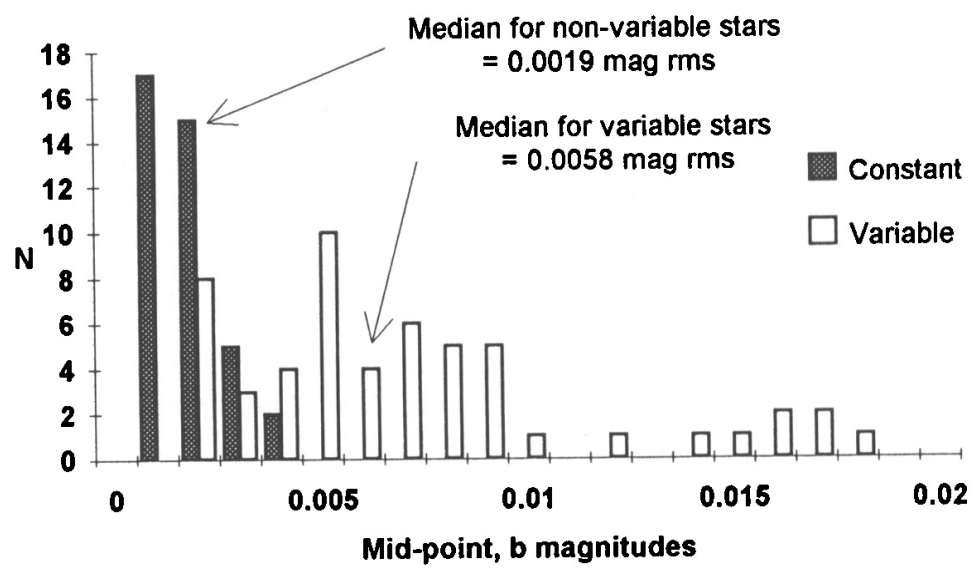

FIgURE 4. Distribution of the dispersion of annual mean $b$ magnitudes over 9 seasons for non-variable and variable stars.

The stars are grouped into trios or quartets comprised of one or two program stars and two adjacent (within a few degrees) comparison stars of similar color and visual magnitude. A nightly observation takes about $1 / 2$ hour and involves cycling through the group two times through each of two filters, $b(472 \mathrm{~nm})$ and $y(551 \mathrm{~nm})$. This produces four independent sets of differential magnitudes. We typically observe each group about 10 times per season.

From observations on 1000 nights over the past decade, we have mapped out the slow year-to-year variations, which range from $0.1-3 \%$ rms (Skiff \& Lockwood 1986; Lockwood \& Skiff 1988, 1990). The variations tend to be smooth rather than random, and some appear to be cyclic. The amplitude of variation decreases with increasing stellar age, just as chromospheric activity does (Lockwood et al. 1992). Despite an impressive improvement in precision over the Lowell study three decades ago, we are still unable, by a factor of three or so, to detect variations in stars as small as the $0.1 \%$ peak-to-peak change in total solar irradiance seen in cycle 21 (Willson \& Hudson 1991). Nevertheless, useful comparisons are possible. 

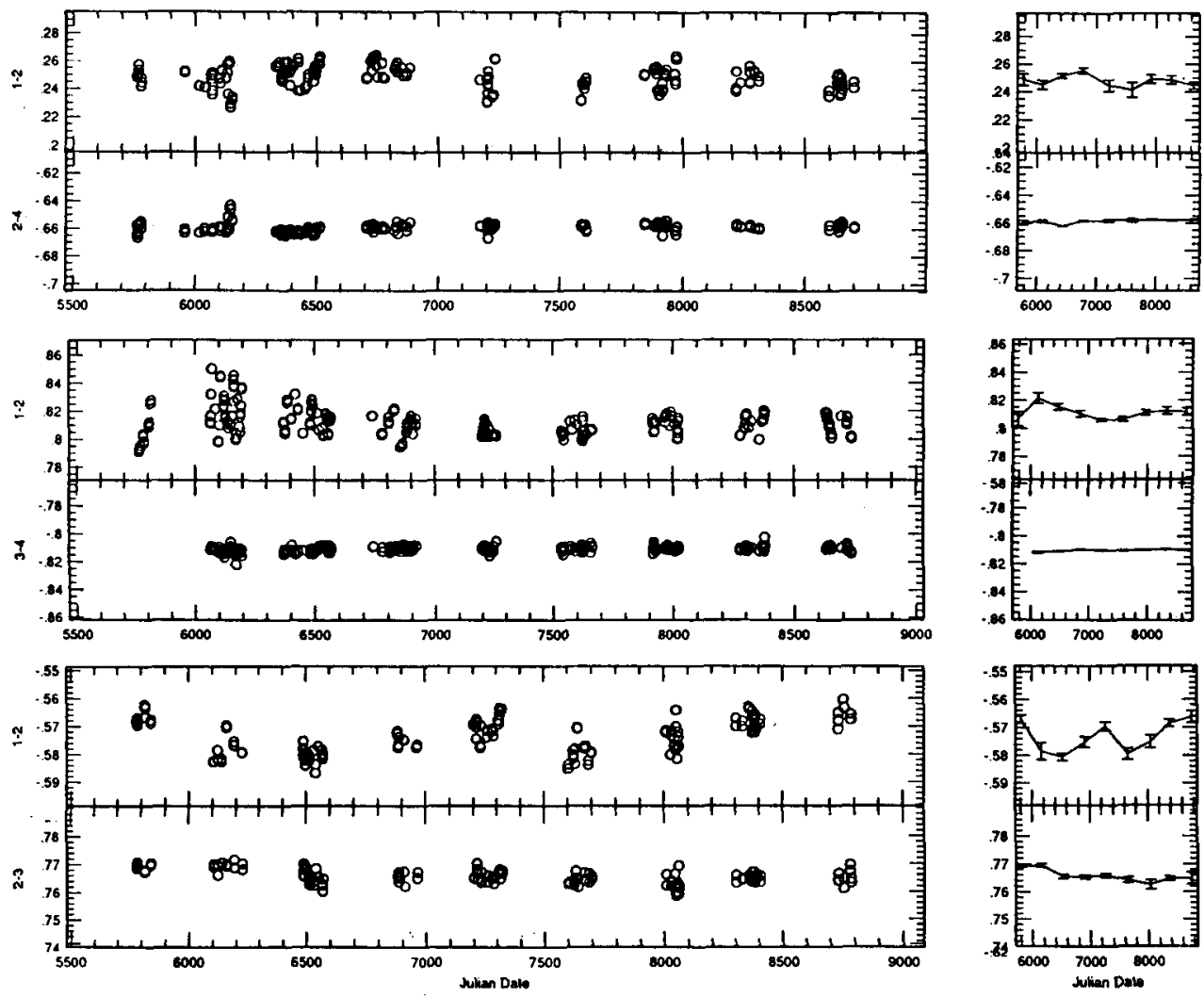

Figure 5. Light curves ( $b$ and $y$ averaged) for three stars whose variations appear cyclic. Below each differential light curve we show the corresponding light curves for the comparison star pair. The significance of the variation can therefore be judged by comparing the two light curves. (left) the individual nightly data points. (right) annual mean magnitudes and $95 \%$ confidence intervals. The program stars are: (top) HD35296; (center) HD82885; (bottom) HD115383. The comparison star pair for HD115383 show a secular trend, so one of these stars must also be variable. HD82885 showed strong rotational modulation during the second observing season, accounting for the large scatter in that year (Skiff \& Lockwood 1986).

\section{Observational errors}

Owing to skill and attentiveness to sky conditions, the observer (Brian A. Skiff) seldom makes a measurement that must be rejected later. Nonetheless, acceptable nights vary in quality and some observations are better than others. The histograms of measurement dispersion (Figure 2) show the distribution of error over half-hour observing intervals.

Two points are noteworthy: (1) the distribution of noise is virtually identical in $b$ and $y$. It arises in similar proportions from scintillation, Poisson noise, sky transparency variations, and guiding errors (cf. Table 2 in Lockwood \& Skiff 1988). (2) The median dispersion is $0.002 \mathrm{mag} \mathrm{rms}$ and few observations are worse than $0.010 \mathrm{mag}(1 \%)$. The tail of the distribution comes mostly from observations on poor nights. Assuming four observations per night (two each in $b$ and $y$ ) and $10+$ nights per season, the predicted observational error in annual mean magnitudes is $<0.001 \mathrm{mag}$. 

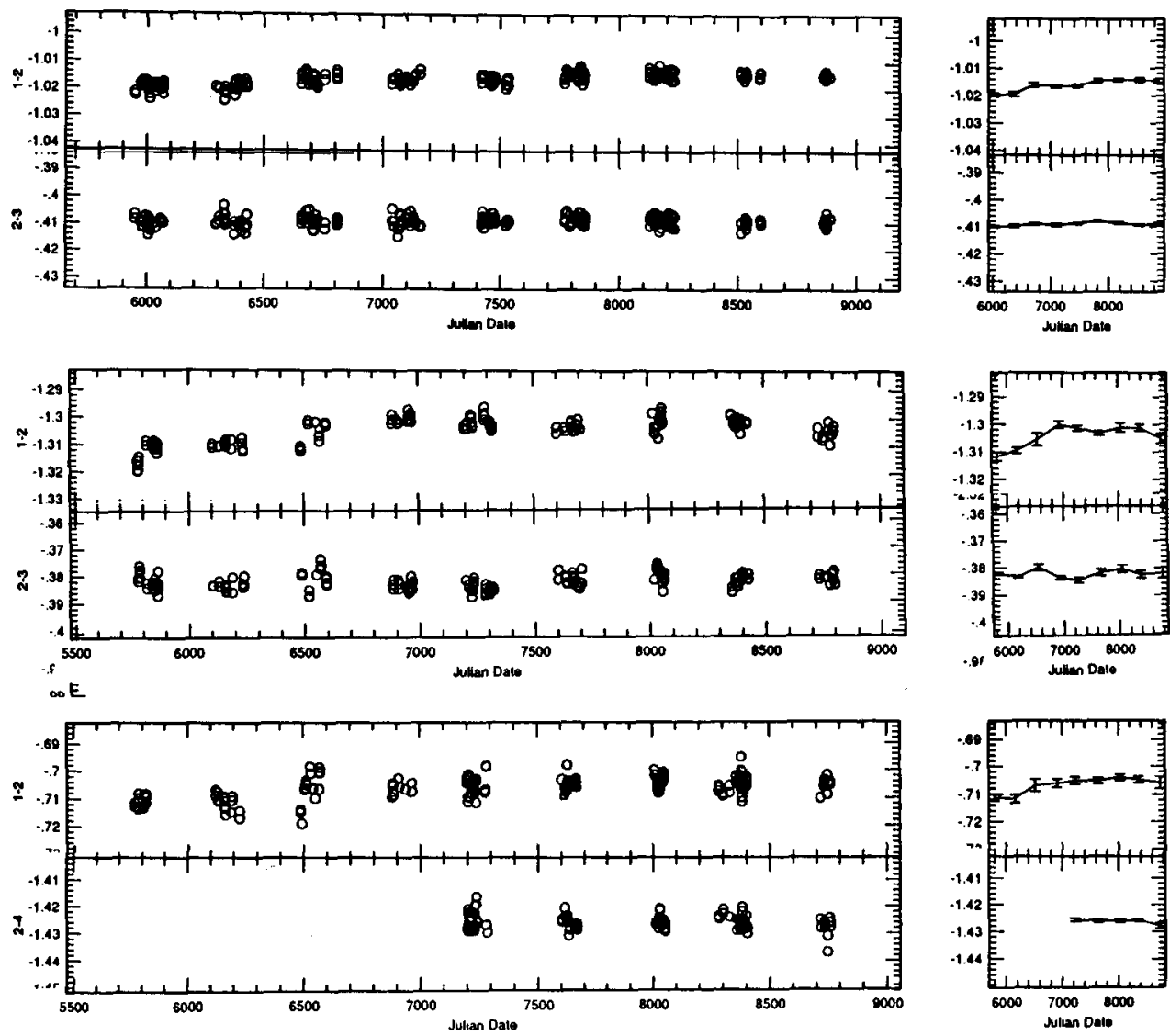

FiguRE 6. Light curves for three stars whose brightness varied monotonically (top) HD10476; (middle) HD120136; (bottom) HD114710. The mean chromospheric activity levels of these three stars are only slightly higher than the Sun's. HD10476 is a close solar analog with a distinct $~ 10$ year chromospheric activity cycle (see paper by Radick, this volume). One of the comparison stars for HD120136 is unstable; it may be a $\delta$ Scuti variable.

\section{Detecting variable stars}

Observing stars in trios (or quartets) yields three (or six) parallel time series of differential magnitudes for pairwise star combinations. By testing the significance of the correlation between pairs of light curves, we determine which stars in a group are variable. Sometimes a comparison star is variable-an unfortunate but not unexpected complication-hence the need for multiple comparison stars. The threshold of detectable variability for a program star depends critically on the inherent stability of its comparison stars, a property which differs from group to group. The best comparison stars are stable to $<0.001$ mag.

Corroborating evidence for variability comes from the tendency of variability to recur season after season. Further, because of the blackbody distribution of stellar radiation, intrinsic variations in $b$ are greater than in $y$, while observational error shows no such color dependence.

Figure 3 shows histograms of magnitude dispersion over the duration of single observing seasons (months) for variable and non-variable stars. The histograms overlap because of 


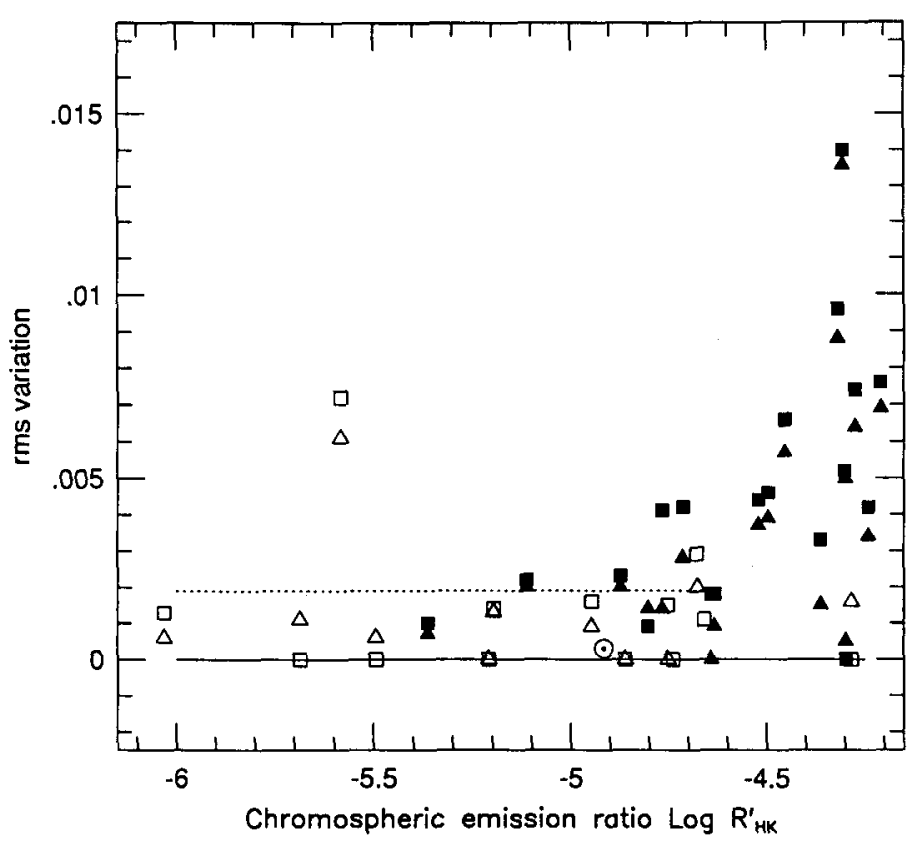

Figure 7. The rms dispersion of annual mean magnitudes, $b$ (squares) and $y$ (triangles), as a function of the chromospheric emission ratio, $\log R_{\mathrm{HK}}^{\prime}$. Filled symbols indicate stars whose intrinsic variability is decisively established by statistical tests. Open symbols indicate stars whose observed fluctuations do not exceed the fluctuations of their particular comparison stars. The star near $(-5.6,0.007)$ may be variable but its comparison star pair is so unreliable that no decision is possible. The active star HD129333 is omitted because its large variations lie off scale. The dotted line indicates the median value of measurement error and intrinsic variability for comparison stars. Plotted values falling below this line have been significantly adjusted and represent, for those stars which are not demonstrably variable, upper limits to intrinsic variability.

differing thresholds of detectable variability from group to group. For non-variable stars, the median dispersion is $0.0019 \mathrm{mag}$. Since the median dispersion for detected variable stars is more than twice this large, the distinction between variables and non-variables is often clear cut. Under the most favorable circumstances when the comparison stars are exceptionally stable, we can detect short-term variability as small as $\sim 0.0015 \mathrm{mag} \mathrm{rms}$.

Similarly, in Figure 4 we show the distribution of the dispersion of annual mean magnitudes over the 9-year duration of the program. The median value for non-variable stars was only $0.0019 \mathrm{mag}$, and the smallest detected variation was $0.0015 \mathrm{mag}$.

\section{Some light curves}

The two principal data products from our program are light curves, which display data in an easily comprehended visual format, and formal statistics quantifying the amount and significance of variability. Figures 5 and 6 show some sample light curves for stars showing evidence of photometric cycles and monotonic trends, respectively. The light curves display individual nightly points on the left-hand panels and annual mean brightness levels with $95 \%$ confidence error bars on the right-hand panels. To simplify the presentation we have averaged data for $b$ and $y$. The light curves appear in pairs with 
the top panel showing the differential magnitude for program star minus comparison star ("signal") and the lower panel showing analogous data for the comparison stars ("noise").

The average levels of chromospheric activity for the three stars in Figure 5 are all higher than the Sun's. Their light variations are correlated inversely with $\mathrm{Ca}$ II H and $\mathrm{K}$ variation as is typical for young, active stars (Lockwood et al. 1992).

Figure 6 shows three stars whose annual mean brightness changed monotonically over nine years.

\section{Photometric variability and mean chromospheric activity}

We found previously that photometric variability is a strong function of mean chromospheric variability, and hence, stellar age (e.g., Lockwood \& Skiff 1990; Lockwood et al. 1992). Figure 7 shows an updated version of the relationship between the rms dispersion in annual mean $b$ and $y$ magnitudes and the chromospheric emission ratio, $\log \mathrm{R}_{\mathrm{HK}}^{\prime}$. This ratio, expressed in the notation of Noyes et al. (1984) but calculated using a different prescription for correcting for the non-magnetic component of the emission, estimates the fraction of a star's output arising from the chromosphere. Different symbols indicate the presence or absence of detected photometric variability. The intrinsic stellar variability is almost always greater in $b$ than in $y$. A regression of $\sigma_{b}$ on $\sigma_{y}$ yields $\sigma_{b} / \sigma_{y}=1.07 \pm 0.03$.

The plotted values in Figure 7 , have been adjusted slightly downward to correct for measurement errors and variability of the comparison stars. We subtracted the observed variance of the comparison star magnitudes, or in a few cases where the comparison stars are clearly suspect, we used estimated typical values. In most cases the change is trivial, but it does make a difference for some stars whose variability is either small or undetectable, that is, those on the left side of the diagram. For them, the values plotted represent a statistically unbiased estimate of the upper limit of possible intrinsic variability.

We included the Sun in Figure 7 based on its observed variation from 1980-1989 (Willson \& Hudson 1991), adjusting the plotted value slightly upward to account for the distinction between variation in total irradiance and the variation in the visual continuum. Several stars located near the Sun on this diagram are demonstrably variable by much larger amounts than the Sun, a factor of four on the average. Thus, the Sun's recent photometric variability appears deficient relative to some similar stars.

In reaching this conclusion we recognize several deficiencies that can be at least partially remedied by further solar and stellar observations. Foremost and most easily rectified is the limited duration of present data-just over one 11-year cycle for the Sun and probably less than a cycle length for many of the stars. We know that our photometric observations may not sample the full range of chromospheric variation, as shown for example in the illustrations in the paper by Radick in this volume. It seems equally likely that the full range of solar irradiance variation over activity cycles of differing amplitude is not exhibited in the available data. Secondly, as solar variability lies well below the level at which we can reliably detect stellar variability, we can only point to those stars whose variability grossly exceeds that of the Sun as examples of what the Sun's behavior might be like at some other time. We have no real population statistics to lead us to a picture of normal stellar behavior, only the tantalizing indication that the Sun has potential for greater variations that we have observed thus far. 


\section{REFERENCES}

Аввотт, C. G. 1963 Solar Variation and Weather. Smithsonian Misc. Coll. 146, No. 3.

Baliunas, S. L., \& JASTRow, R. 1990 Evidence for long-term brightness changes of solar-type stars. Nature 345, 520-522.

Baliunas, S. L. \& Vaughan, A. H. 1985 Stellar activity cycles. Ann. Rev. Astron. \& Astrophys. 23, 379 .

Clayton, H. H. 1923 Solar Relations to Weather and Life. The Clayton Weather Service: Canton, MA.

Huntington, E. 1923 Earth and Sun. Yale University Press: New Haven.

Jerzykiewicz, M., \& Serkowski, K. 1966 The Sun as a Variable Star. Lowell Observatory Bulletin (No. 137) 6, 295-323.

Lockwood, G. W., \& SKIFF, B. A. 1988 Luminosity variations of stars similar to the Sun. Air Force Geophysics Lab Report AFGL-TR-88-0221, 101 pp. AFGL: Hanscom Air Force Base.

LOCKWOOD, G. W. \& SKIFF, B. A. 1990 Some insights on solar variability from precision stellar astronomical photometry. In Climate Impact of Solar Variability (ed. K. H. Schatten \& A. Arking). NASA Conference Publ. 3086. pp. 8-15.

Lockwood, G. W., Skiff, B. A., Baliunas, S. L., \& Radick, R. 1992 Long-term solar brightness changes estimted from a survey of Sun-like stars. Nature 360, 653-655.

McCormac, B. M. 1983 Weather and Climate Responses to Solar Variation. Colorado Associated University Press: Boulder.

Noyes, R. W., Hartmann, L. W., Baliunas, S. L., Duncan, D. K., \& Vaughan, A. H. 1984 Rotation, convection, and magnetic activity in lower main-sequence star. Astrophys. J. 279, 763-777.

Radick, R. R., Lockwood, G. W., Thompson, D. T., Duncan, D. K., \& BaggetT, W. E. 1987 The activity, variability, and rotation of lower main-sequence Hyades stars. Astrophys. J. 321, 459-472.

Schatten, K. H., \& ARking, A. ED. 1990 Climate Impact of Solar Variability. NASA Conference Publ. 3086.

SKIFF, B. A., \& LockwOoD, G. W. 1986 The photometric variability of solar-type stars. V. The standard stars 10 and 11 Leonis Minoris. Publ. Astron. Soc. Pacific 98, 338-441.

Willson, R. C. \& Hudson, H. S. 1991 The Sun's luminosity over a complete solar cycle. Nature 351, 42-44.

Wilson, O. C. 1978 Chromospheric variations in main-sequence stars. Astrophys. J. 226, 379396. 